\title{
ANÁLISE TEMPORAL DA DISPONIBILIDADE HÍDRICA NOS ESTADOS BENEFICIADOS PELA TRANSPOSIÇÃO DO RIO SÃO FRANCISCO
}

\author{
Higor Costa de Brito \\ Universidade Federal de Campina Grande - UFCG \\ Centro de Tecnologia e Recursos Naturais, Campina Grande, PB, Brasil \\ h igor@hotmail.com \\ Yáscara Maia Araújo de Brito \\ Universidade Federal de Campina Grande - UFCG \\ Centro de Tecnologia e Recursos Naturais, Campina Grande, PB, Brasil \\ yascaramaiaa@gmail.com \\ Wanessa Dunga de Assis \\ Universidade Federal de Campina Grande - UFCG \\ Centro de Tecnologia e Recursos Naturais, Campina Grande, PB, Brasil \\ w dunga@hotmail.com \\ Yuciara Costa Barbosa Ferreira \\ Universidade Federal de Campina Grande - UFCG \\ Centro de Tecnologia e Recursos Naturais, Campina Grande, PB, Brasil \\ yuciara.barbosa@gmail.com \\ Rochele Sheila Vasconcelos \\ Universidade Federal de Pernambuco \\ Centro Acadêmico do Agreste, Caruaru, PE, Brasil \\ rochelly17@hotmail.com \\ Iana Alexandra Alves Rufino \\ Universidade Federal de Campina Grande - UFCG \\ Centro de Tecnologia e Recursos Naturais, Campina Grande, PB, Brasil \\ iana.alexandra@ufcg.edu.br
}

\begin{abstract}
RESUMO
O Projeto de Integração do Rio São Francisco foi elaborado a fim de reduzir os impactos das secas nos estados do Semiárido Brasileiro. O artigo realiza uma análise temporal da disponibilidade hídrica nos quatro estados receptores das águas do Projeto de Integração do Rio São Francisco (PISF) através da tabulação cruzada de dados referentes à superfície hídrica e a precipitação anual dos estados no período de 1985 a 2017. Seu principal objetivo consiste na identificação da correlação entre esses dois parâmetros e as inferências que podem ser feitas a partir dos resultados nos 33 anos de análise. A metodologia consiste em determinar a correlação existente entre as áreas classificadas como superfície hídrica pelo projeto MapBiomas e índices pluviométricos obtidos por satélite. São utilizados também dados referentes à recorrência hídrica do produto Global Water Surface para validar a discussão acerca da disponibilidade de água. Os resultados confirmam a seca plurianual ocorrida desde 2012 e a consequente perda significativa de superfície hídrica dos estados, além de identificar um aumento da correlação desse fator com a diminuição da precipitação anual nos estados da Paraíba, Pernambuco e Rio Grande do Norte a partir da década de 90 . Os dados de recorrência destacam a fragilidade hídrica dos estados e evidencia a constante deficiência de precipitação para atender às demandas existentes. Por conseguinte, salienta-se a necessidade da implantação de medidas de gestão efetivas a fim de garantir o desenvolvimento regional sustentável e assegurar a disponibilidade hídrica do Rio São Francisco e de suas bacias receptoras.
\end{abstract}

Palavras-chave: Superfície hídrica. Precipitação. Uso do solo. Seca. Sensoriamento remoto.

\section{TEMPORAL ANALYSIS OF WATER AVAILABILITY IN THE STATES BENEFITED BY THE TRANSPOSITION OF THE SÃO FRANCISCO RIVER}

\section{ABSTRACT}

The São Francisco River Integration Project was designed to reduce the impacts of droughts in the states of Brazilian semiarid. The article performs a temporal analysis of the water availability in the four PISF water receiving states, through the cross-tabulation of data 
referring to the water surface and the annual precipitation of the states in the period from 1985 to 2017. To identify the correlation between these two parameters and inferences that can be made from the results in the 33 years of analysis. The methodology consists in determining the correlation between the areas classified as water surface by the MapBiomas project and rainfall obtained by satellite. Water recurrence data from the Global Water Surface product are also used to validate the discussion about water availability. The results point to the multiannual drought that occurred since 2012 and the consequent loss of water surface of the states, besides identifying an increase of correlation in the states of Paraíba, Pernambuco and Rio Grande do Norte from the 90s. The results highlight the water management fragility of the states, and shows that the precipitation is not enough for the current demands. Therefore, there is a real need of an effective management to ensure sustainable regional development and water availability of the São Francisco River and its sub-basins.

Keywords: Water surface. Precipitation. Land use. Drought. Remote sensing.

\section{INTRODUÇÃO}

As secas são fenômenos naturais e recorrentes que surgem a partir de déficits de precipitação e atinge tanto áreas de alta quanto de baixa pluviosidade. Os impactos causados ocorrem em diferentes setores da sociedade, resultando em danos significativos (materiais e humanos) e perdas (socioeconômicas), nas esferas ambiental, social e econômica (ALVALÁ et al., 2017). Por esse motivo, a gestão desses fenômenos requer a adoção de medidas no âmbito de diferentes políticas públicas setoriais (hídrica, clima, solo).

A classificação das secas depende da duração, intensidade e impactos gerados (CUNHA et al., 2017). Qualquer região apresenta perigo de secas, delineado como um fator natural, entretanto, é a junção do perigo com a vulnerabilidade (este último atrelado a fatores sociais) que determina o risco associado à seca para uma região exposta ao evento, ou seja, a probabilidade e intensidade de ocorrência em vários níveis de severidade (WILHITE, 2012).

No Brasil, a região que apresenta maior risco é o Semiárido Brasileiro (SAB), ao vivenciar uma realidade conflituosa e desafiadora por usos múltiplos dos recursos hídricos, em função da variabilidade climática e da complexa gestão de suas águas. As dificuldades diante das frequentes secas na região causam impactos em áreas urbanas e rurais, nos níveis local, regional e nacional. Duas são as grandes causas propulsoras: a escassez hídrica, seja essa qualitativa ou quantitativa e, a não existência ou inconformidade do gerenciamento dos recursos hídricos (AMORIMet al.. 2016; RÊGO et al., 2015).

Como alternativa à redução dos riscos foi elaborado o Projeto de Integração do Rio São Francisco (PISF) com bacias do Nordeste Setentrional em dois eixos de ligação, o eixo Norte e o eixo Leste. As águas são captadas no estado de Pernambuco, na calha do rio principal, entre as barragens de Sobradinho e Itaparica e são destinadas ao abastecimento de bacias hidrográficas em quatro unidades federativas: Ceará, Paraíba, Pernambuco e Rio Grande do Norte (BRASIL, 2004).

O artigo realiza uma análise temporal da disponibilidade hídrica nos quatro estados receptores das águas do PISF, através da tabulação cruzada de dados referentes à superfície hídrica e a precipitação anual dos estados no período de 1985 a 2017. O objetivo principal é identificar a correlação entre esses dois parâmetros e as inferências que podem ser feitas a partir dos resultados nos 33 anos de análise.

\section{REVISÃO DE LITERATURA}

O primeiro registro de seca anual no Nordeste brasileiro data do início do período de colonização, em 1583, século XVI. No entanto, com a ocupação progressiva da região, as secas registradas passaram a ser também plurianuais, com registros de até 7 anos, nas quais, os impactos sociais e econômicos se tornaram mais intensos (CAMPOS, 2014; MARTINS et al., 2015).

A primeira seca plurianual foi identificada no período de 1721 a 1727 e afetou os estados do Ceará, Rio Grande do Norte, Paraíba e Pernambuco. Em outro registro, o período de 1877 a 1879 apontou para os maiores impactos até então, que desestruturou a população nordestina, dizimou quase que completamente o rebanho bovino e provocou o êxodo rural de aproximadamente metade da população para áreas consideradas mais prósperas (MAISONNAVE e PRADO, 2018). 
Segundo Martins et al. (2015, p. 11) "os primeiros registros são realizados em função dos impactos a elas associados, enquanto as mais recentes são baseadas em observações meteorológicas". As secas identificadas no século XXI, não só no Brasil, são caracterizadas por temperaturas mais altas, duração mais prolongada e maior extensão espacial, influenciadas diretamente pelas demandas por água (CRAUSBAY e RAMIREZ, 2017).

No final do século XX e início do XXI os maiores períodos de seca ocorreram entre 1979-1983 e 1990-1993, com registros também em 1998-1999, 2001 e 2003. Esses eventos foram marcados pelo desenvolvimento de ações para o combate à seca, a exemplo da criação da SUDENE (Superintendência do Desenvolvimento do Nordeste) na década de 80, que impulsionou a construção de reservatórios para o aumento da oferta hídrica (MAISONNAVE e PRADO, 2018).

Dentre os casos recentes tem-se a maior seca já registrada na região Nordeste, iniciada em 2012 e que vem causando inúmeros impactos: açudes entraram em colapso, com volumes zerados; diversos municípios passaram a depender de abastecimento por carros-pipa; a irrigação foi proibida em muitas regiões e a agropecuária apresentou perdas críticas (MARENGO et al., 2016).

No SAB as secas não ocorrem de forma homogênea. A seca total apresenta efeitos em todas as áreas da região atingida, enquanto as secas parciais alcançam apenas algumas áreas. Entretanto, todos os impactos são significativos e contribuem para o agravamento do fenômeno de desertificação no Nordeste (TAVARES et al., 2019). Um exemplo desses impactos está no estado da Paraíba que, entre 2017 e 2018, decretou estado de emergência para 196 dos seus municípios ( $88 \%$ das localidades totais), devido à escassez hídrica provocada pela seca iniciada em 2012 (PARAíBA, 2018). Situação similar foi percebida nos outros estados da região.

A vulnerabilidade às secas do $S A B$ está condicionada aos fenômenos de interação oceano-atmosfera nos Oceanos Pacífico e Atlântico da região tropical do globo terrestre e às fases quente (El Niño) e fria (La Niña) do fenômeno climático global El Niño-Oscilação Sul (ENOS), que favorecem ou desfavorecem as atividades convectivas, principalmente a posição e intensidade da Zona de Convergência Intertropical (ZCIT) (COELHO et al., 2002; DE SOUZA et al., 2005). Em anos de La Niña, ocorrem anomalias positivas de precipitação em decorrência das perturbações, nos quais a ZCIT posiciona-se ao sul da posição climatológica. Em contraposto, em anos de El Niño há a supressão dos mecanismos de convecção que produzem chuvas, e a ZCIT é deslocada para o norte, tornando a precipitação mais escassa (GRIMM, 2003, 2004; MEDEIROS et al., 2019; TIMMERMANN et al., 2018).

Os baixos índices pluviométricos (inferiores a $900 \mathrm{~mm}$ ), as temperaturas elevadas durante todo ano com baixas amplitudes térmicas (entre $2^{\circ} \mathrm{C}$ e $3^{\circ} \mathrm{C}$ ), a forte insolação, as altas taxas de evapotranspiração (2.500 $\mathrm{mm}$ ao ano) e a irregularidade do seu regime contribuem para a baixa disponibilidade hídrica do Semiárido. Assim, os elevados índices de evapotranspiração comumente superam os totais pluviométricos irregulares, resultando em taxas negativas no balanço hídrico. $O$ Nordeste Setentrional, que inclui o Ceará, Pernambuco, Rio Grande do Norte e Paraíba, é o mais susceptível (ANA, 2017; MONTENEGRO e MONTENEGRO, 2012).

A maioria dos rios do Nordeste Setentrional são intermitentes, ou seja, secam durante os meses sem precipitação. Assim, a garantia de disponibilidade hídrica para o atendimento dos múltiplos usos é obtida através da construção de açudes. São esses reservatórios que realizam o abastecimento público humano de aproximadamente $70 \%$ da população, por exemplo. Além de servirem também como principal fonte para a irrigação dessas regiões. O PISF objetiva aumentar a garantia da oferta de água nesses açudes (ANA, 2017).

Por isso, a necessidade de se acompanhar e mapear a superfície hídrica nessa região se torna primordial. Esse mapeamento, a longo prazo, através do monitoramento por métodos tradicionais de medições in situ, torna-se bastante complexo, principalmente em ambientes heterogêneos e de grande extensão (NORMANDIN et al., 2018). Em contrapartida, o Sensoriamento Remoto (SR) fornece dados substanciais de fácil acesso para o acompanhamento da situação dos recursos hídricos. As técnicas de SR são capazes de realizar medições contínuas e atualizadas da cobertura terrestre em escala global, podendo contar com observações terrestres para desenvolvimento e validação de algoritmos (TANG et al., 2009; WANG e XIE, 2018).

Além da análise da cobertura, essa técnica também é capaz de estimar índices pluviométricos a partir de dados infravermelho coletados por satélites, podendo fornecer informações abundantes e com alta resolução espaço-temporal sobre regiões extensas, onde os dados de precipitação são escassos ou inexistentes (PAREDES-TREJOAR et al., 2017). 
Higor Costa de Brito

Yáscara Maia Araújo de Brito

Wanessa Dunga de Assis

Análise temporal da disponibilidade hídrica nos estados beneficiados pela transposição do Rio São Francisco

Yuciara Costa Barbosa Ferreira Rochele Sheila Vasconcelos lana Alexandra Alves Rufino

O sensoriamento remoto tem demonstrado um grande potencial na detecção e monitoramento de superfícies hídricas ao longo de grandes recortes temporais (ALSDORF et al., 2007; SMITH, 1997), ao fornecer dados multiespectrais e multitemporais de baixo custo, além de detectar informações valiosas para a compreensão e monitoramento de padrões e processos de desenvolvimento da superfície terrestre (WENG, 2002).

\section{MATERIAL E MÉTODO}

O percurso metodológico do estudo (Figura 1) foi dividido em duas etapas: a primeira, caracterizada pela aquisição dos dados de sensoriamento remoto; e a segunda, correspondente à manipulação desses produtos para a análise quali-quantitativa. Os procedimentos foram realizados para o recorte temporal de 1985 a 2017 e recorte espacial correspondente aos quatro estados do Nordeste Setentrional beneficiados pelo PISF.

Figura 1 - Fluxograma metodológico.

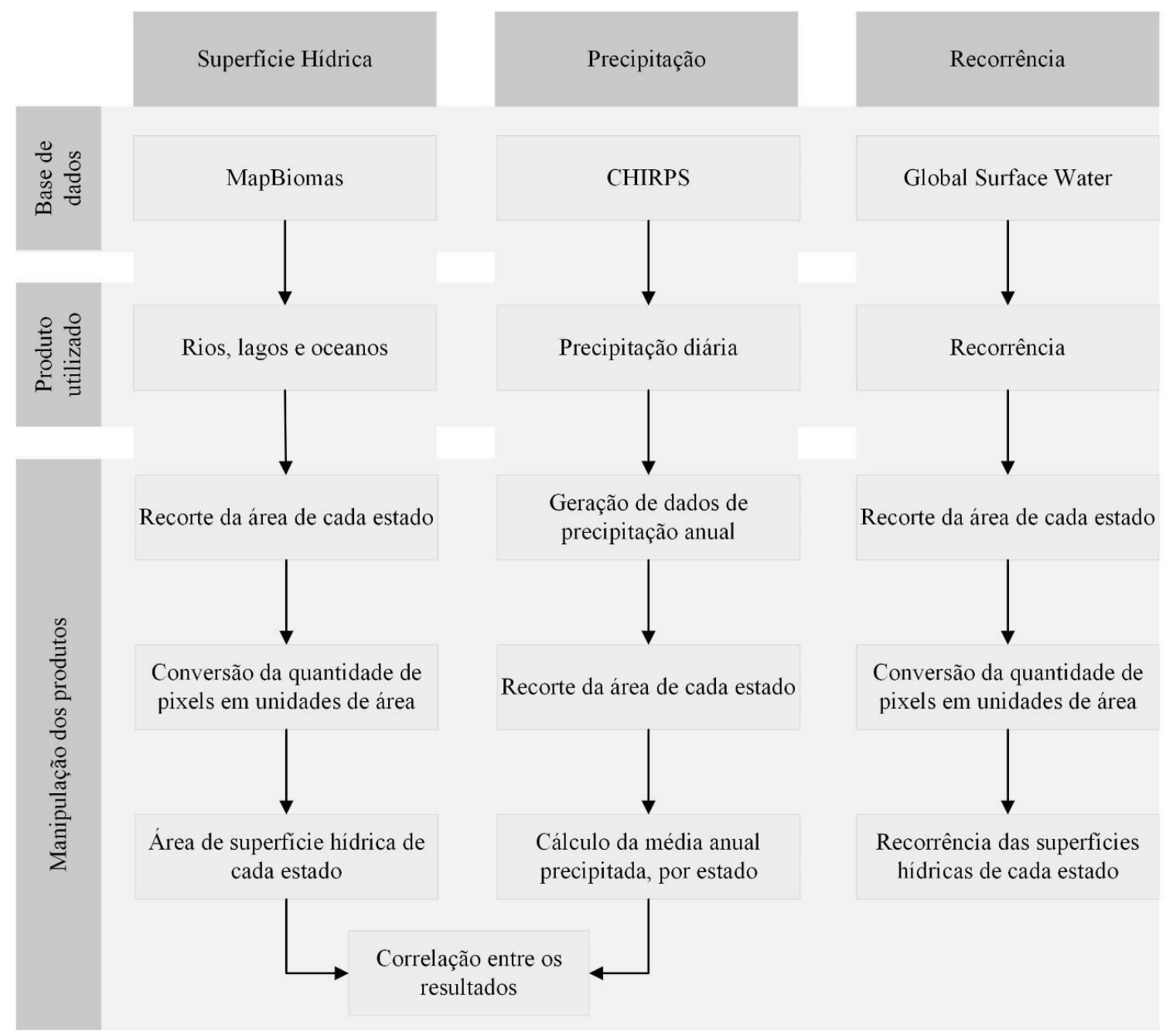

Fonte: Autoria própria.

\section{CARACTERIZAÇÃO DA ÁREA DE ESTUDO}

A área de estudo consiste em quatro estados do Nordeste brasileiro, mais especificamente os que formam o Nordeste Setentrional - Ceará, Paraíba, Pernambuco e Rio Grande do Norte (Figura 2). A escolha justifica-se por serem os estados que contém as bacias receptoras das águas provenientes do PISF. Esse projeto visa garantir segurança hídrica ao Agreste e Sertão dos estados citados, que

$\begin{array}{lllll}\text { Caminhos de Geografia } \quad \text { Uberlândia-MG } & \text { v. 21, n. } 75 \quad \text { Jun/2020 } & \text { p. 102-116 Página } 105\end{array}$


Higor Costa de Brito

Yáscara Maia Araújo de Brito

Wanessa Dunga de Assis

Análise temporal da disponibilidade hídrica nos estados beneficiados pela transposição do Rio São Francisco

Yuciara Costa Barbosa Ferreira Rochele Sheila Vasconcelos lana Alexandra Alves Rufino

correspondem a 12 milhões de pessoas, em 390 municípios reconhecidos como vulneráveis às secas. A obra divide-se em dois trechos: o eixo Leste, em funcionamento desde 2017, tem 217 quilômetros de extensão, e beneficia a bacia do rio Paraíba (PB) e a bacia do rio Moxotó (PE); O eixo Norte, por sua vez, tem 260 quilômetros de extensão e como destino as bacias do rio Apodi (RN) e do rio Piranhas-Açu (PB/RN), o Sistema Jaguaribe-Metropolitano (CE) e as bacias dos rios Brígida e Terra Nova (PE) (BRASIL, 2004).

Figura 2 - Caracterização da área de estudo.
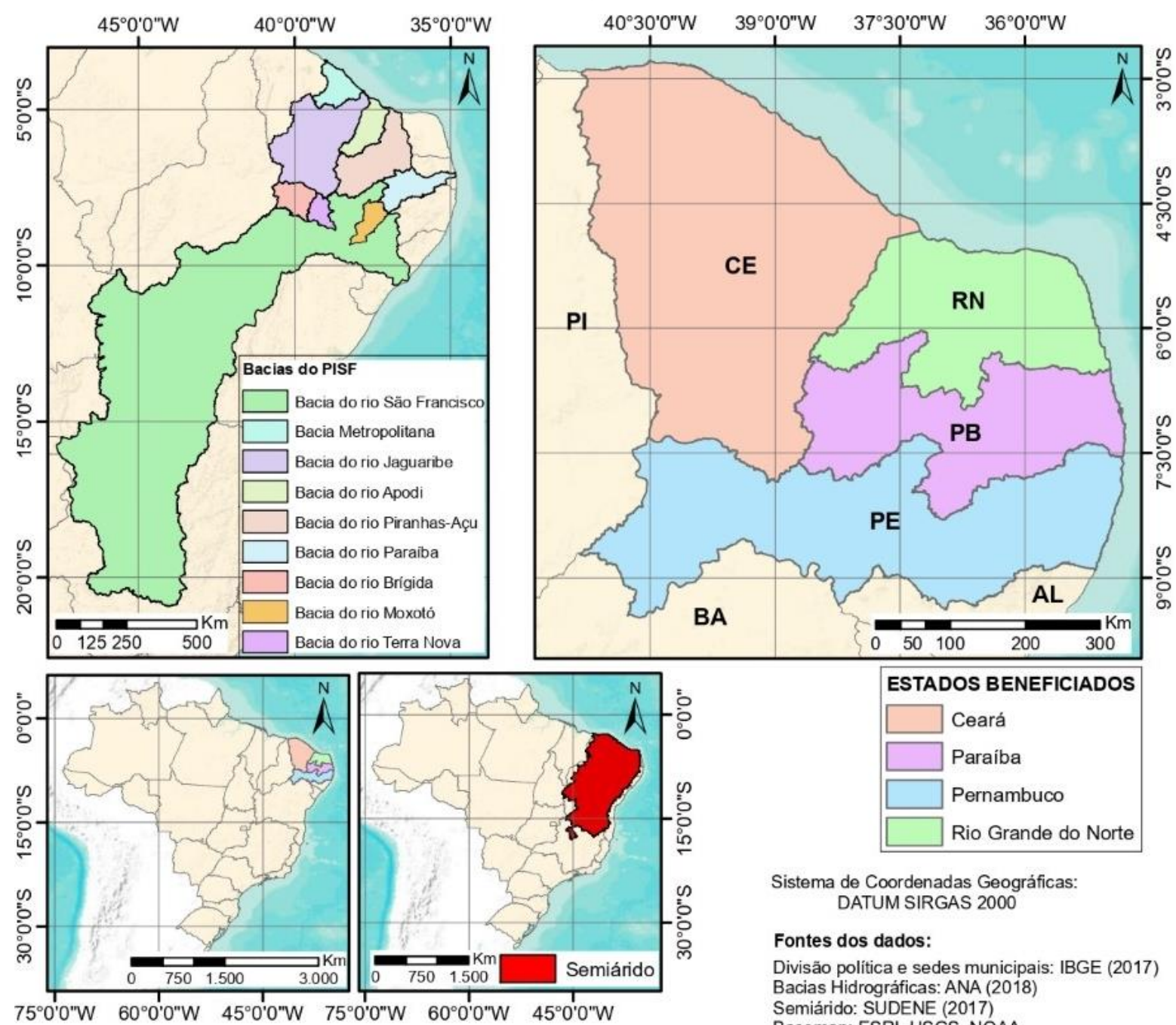

ESTADOS BENEFICIADOS

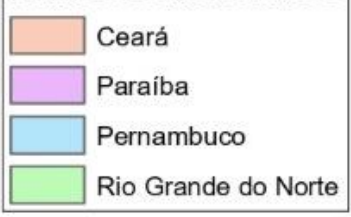

Sistema de Coordenadas Geográficas: DATUM SIRGAS 2000

Fontes dos dados:

Divisão politica e sedes municipais: IBGE (2017) Bacias Hidrográficas: ANA (2018)

Semiárido: SUDENE (2017)

Basemap: ESRI, USGS, NOAA

Fonte: Elaborada a partir de IBGE (2017a), SUDENE (2017) e ANA (2019b).

Ademais, é necessária também a caracterização dos estados em análise quanto à disponibilidade de água superficial existente. A Figura 3 é proveniente da plataforma Olho N'água e retrata a situação dos volumes de água disponíveis (no mês julho/2019) nos estados analisados no recorte espacial que faz intersecção com o Semiárido Brasileiro.

O Semiárido Potiguar encontra-se na melhor situação dentre os demais, com $23,8 \%$ da sua capacidade total de armazenamento. Em seguida, tem-se o Semiárido Paraibano com quase 19,1\%, o Cearense com $15,8 \%$ e, por fim, o Pernambucano, com apenas $12,1 \%$ de suas reservas superficiais ocupadas para atender a quase 9 milhões de pessoas (INSA, 2019). Destaca-se que, a plataforma não monitora todos os reservatórios de água existentes, todavia, monitora os de maior importância e consequentemente de maior capacidade de armazenamento. 
Figura 3 - Disponibilidade hídrica do Semiárido brasileiro.

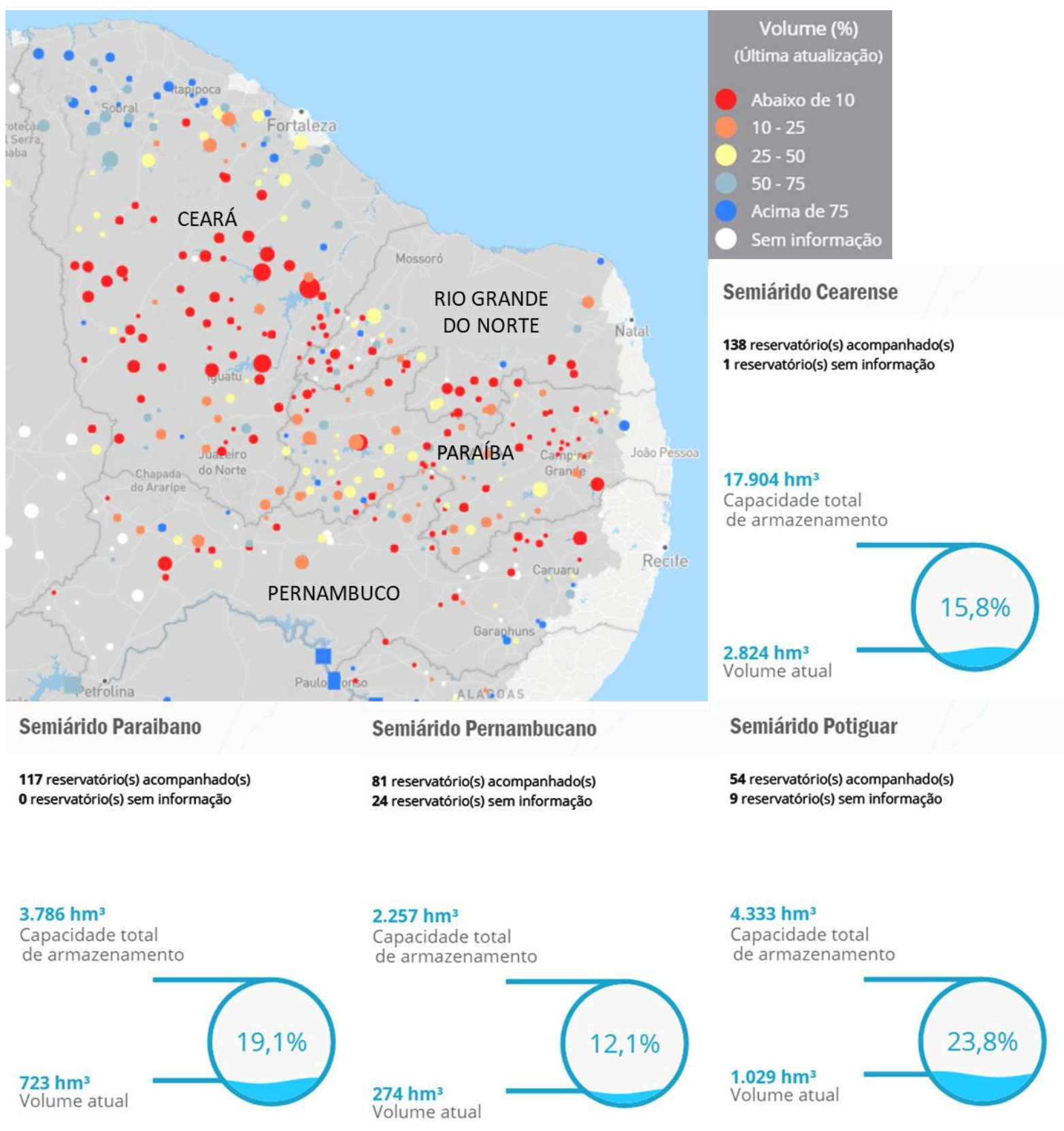

Fonte: Elaborado a partir de dados do INSA (2019).

Na Tabela 1 consta a caracterização dos estados, com as respectivas áreas e o número de habitantes de acordo com o Censo 2010 do IBGE, além da precipitação média anual e o valor correspondente ao ano de menor média precipitada no recorte temporal em análise. $O$ estado de maior área é o Ceará, mas o mais populoso é Pernambuco, com quase 9 milhões de habitantes, seguido da Paraíba, e por último o Rio Grande do Norte (IBGE, 2017b).

As precipitações médias anuais foram calculadas pela média aritmética da precipitação acumulada para o recorte temporal 1985-2017 nos quatro estados. Os valores obtidos são próximos - entre 700 e 800 mm/ano. O ano de 1993 apresentou a menor precipitação média anual para todos os estados com exceção do Ceará, que teve o seu pior ano em 2012 (FUNK et al., 2015). 
Análise temporal da disponibilidade hídrica nos estados beneficiados pela transposição do Rio São Francisco
Higor Costa de Brito

Yáscara Maia Araújo de Brito Wanessa Dunga de Assis

Yuciara Costa Barbosa Ferreira Rochele Sheila Vasconcelos lana Alexandra Alves Rufino

Tabela 1 - Dados referentes aos estados beneficiados.

\begin{tabular}{ccccc}
\hline Estado & Área $\left(\mathbf{K m}^{2}\right)$ & $\begin{array}{c}\text { População } \\
\text { (hab.) }\end{array}$ & $\begin{array}{c}\text { Precipitação média } \\
\text { anual (mm/ano) }\end{array}$ & $\begin{array}{c}\text { Menor média } \\
\text { anual (mm/ano) }\end{array}$ \\
\hline Ceará & $148.914,39$ & 8.453 .381 & 798,71 & 414,44 \\
Paraíba & $56.468,68$ & 3.766 .528 & 758,02 & 345,20 \\
Pernambuco & $98.152,73$ & 8.796 .448 & 714,10 & 352,60 \\
Rio Grande do Norte & $52.809,25$ & 3.168 .027 & 715,42 & 264,94 \\
\hline
\end{tabular}

Fonte: FUNK et al. (2015); IBGE (2017b).

Por fim, tem-se a caracterização da área de estudo tomando como base mais uma plataforma de dados nacional, o Monitor de Secas brasileiro. E, assim como as demais, é uma fonte secundária que disponibiliza dados mensais referentes às secas no Nordeste do país e em mais dois estados da região Sudeste. Seus produtos resultam da composição de dois indicadores meteorológicos e um hidrológico, sendo eles: SPI - Standardized Precipitation Index (MCKEE et al., 1993), SPEI Standardized Precipitation Evapotranspiration Index (VICENTE-SERRANO et al. 2010) e SRI Standardized Runoff Index (SHUKLA e WOOD, 2008), além de uma série de produtos de apoio (MARTINS et al., 2015).

A Figura 4 expõe três meses de dados disponíveis no portal: julho de 2014, que consiste no primeiro dado que deu origem ao portal; janeiro de 2017, que marcou o mês de secas mais intensas registradas na plataforma para os estados em análise; e maio de 2019, o último mês analisado por este artigo, que demonstra severidades de seca mais amenas na região, em especial nos estados estudados, nos quais a intensidade mais severa identificada foi a Seca Grave.

Figura 4 - Acompanhamento da seca por meio do Monitor de Secas brasileiro.

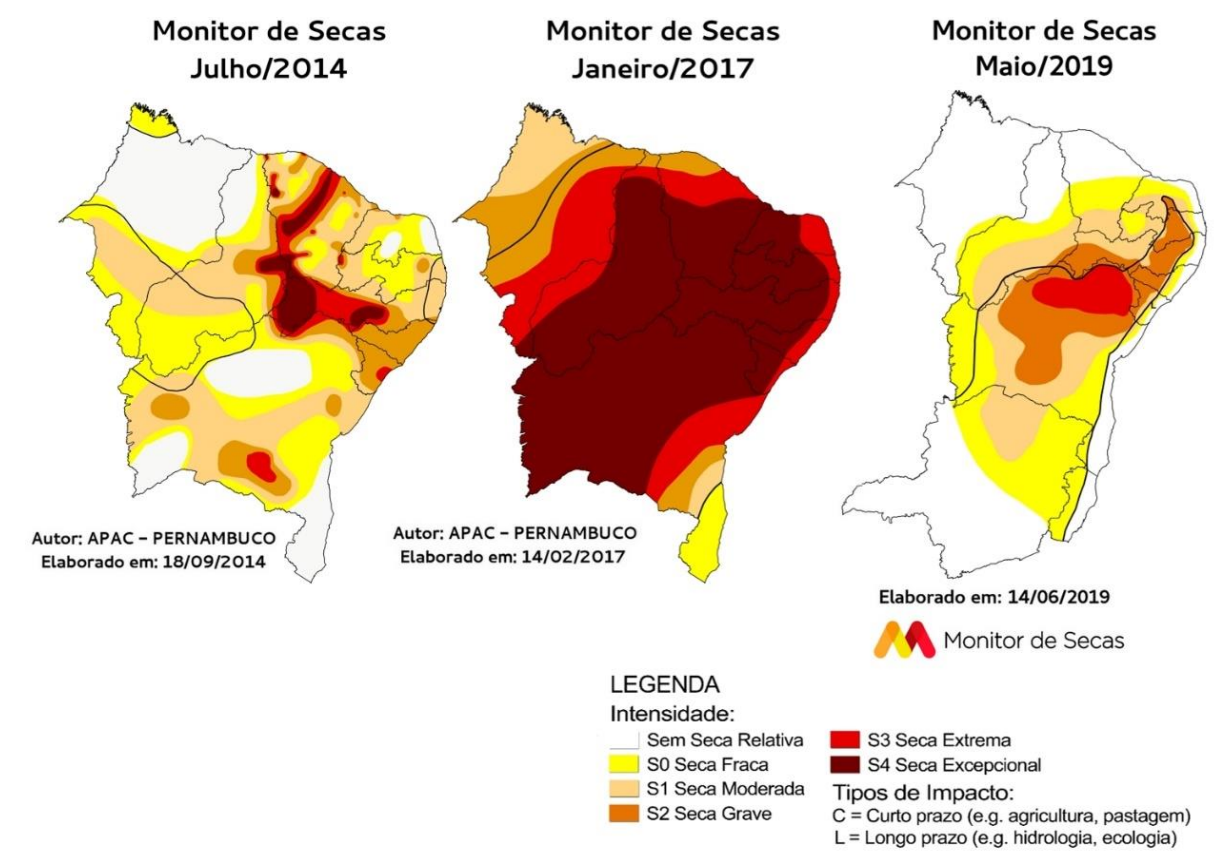

Fonte: Elaborado a partir de ANA (2019a).

\section{AQUISIÇÃO DE DADOS}

Foram utilizados produtos de classificação de uso e ocupação do solo a fim de identificar corpos hídricos superficiais dentro dos limites territoriais dos estados analisados. Para esse fim, utilizou-se os produtos do Projeto de Mapeamento Anual da Cobertura e Uso do Solo do Brasil - MapBiomas (SOUZA e AZEVEDO, 2017), disponibilizados através da plataforma de processamento em nuvem

$\begin{array}{lllll}\text { Caminhos de Geografia } & \text { Uberlândia-MG } & \text { v. } 21, \text { n. } 75 & \text { Jun/2020 } & \text { p. 102-116 Página } 108\end{array}$


Google Earth Engine - GEE (GORELICK et al., 2017), que disponibiliza mapas anuais de classificação de cobertura e uso do solo para todo o território brasileiro com resolução espacial de 30 por 30 metros, elaborados a partir de produtos Landsat. O MapBiomas apresenta 33 mapas anuais de classificação, que compreendem os anos de 1985 a 2017, com perspectiva de atualização contínua.

Para identificar a precipitação anual ocorrida em cada estado foram utilizados dados diários da missão Climate Hazards Group InfraRed Precipitation with Station - CHIRPS (FUNK et al., 2015), também disponibilizados através da plataforma GEE. Os produtos CHIRPS possuem uma resolução espacial de aproximadamente 5,5 por 5,5 quilômetros, fornecendo dados diários a partir do ano de 1981.

Com o intuito de aprimorar a discussão acerca do tema abordado foram utilizados dados de recorrência de corpos hídricos do Global Surface Water - GSW (PEKEL et al., 2016). O GSW é um levantamento de superfícies hídricas globais que disponibilizou diversos produtos referentes ao comportamento de corpos hídricos. Para o presente estudo, utilizou-se o produto de recorrência, que afere a frequência com que a água retorna de ano para ano.

\section{PROCESSAMENTO DOS PRODUTOS}

Os produtos adquiridos foram manipulados através do editor de código da plataforma do GEE, por meio da linguagem de programação javascript, e recortados para o limite de cada estado, para então serem quantificados. Os mapas disponibilizados pelo MapBiomas possuem códigos que remetem a inúmeras classificações de uso e ocupação, dentre eles a classificação "rios, lagos e oceanos".

A quantificação dos produtos MapBiomas foi realizada através de um filtro, que levou em consideração apenas os pixels classificados como "rio, lago ou oceano". Para considerar apenas os rios e lagos, a área litorânea de todos os estados foi desconsiderada, a fim de evitar a contagem de águas oceânicas que pudessem mascarar os resultados. Por fim, a quantificação dos pixels foi convertida em unidades de área, uma vez que cada pixel do produto equivale a uma área de $900 \mathrm{~m}^{2}$.

Os produtos de precipitação diária do CHIRPS foram acumulados para gerar dados de precipitação anual referente aos anos de 1985 a 2017, procedimento este realizado no GEE. Em virtude da heterogeneidade espacial da precipitação, foi feito o cálculo da média precipitada em cada estado, em milímetros por ano, gerando assim um único valor de precipitação para cada ano da série temporal. Os dados de superfície hídrica e precipitação foram agregados em intervalos de décadas (1985-1989; 1990-1999; 2000-2009; 2009-2017) e correlacionados estatisticamente de acordo com a equação abaixo, por estado, com o objetivo de verificar a resposta dos corpos hídricos aos eventos pluviométricos.

$$
\text { Correlação }(X, Y)=\frac{\Sigma(x-\bar{x})(y-\bar{y})}{\sqrt{\sum(x-\bar{x})^{2} \Sigma(y-\bar{y})^{2}}}
$$

Sendo: $x=$ Precipitação acumulada por ano (mm/ano); $y=$ Área de superfície hídrica anual $\left(\mathrm{km}^{2}\right) ; \bar{x}=$ Média da precipitação acumulada no intervalo de décadas (mm/ano); $\bar{y}=$ Média da superfície hídrica no intervalo de décadas $\left(\mathrm{km}^{2}\right)$.

Por fim, o produto de recorrência do GSW é recortado para cada estado e, assim como os produtos de superfície hídrica do MapBiomas, teve a área litorânea dos estados desconsiderada. A quantificação foi exportada como uma planilha de dados, onde a recorrência é classificada percentualmente, com valores variando de 1 a $100 \%$. Dessa forma, pixels com valor 5 referem-se a uma área de superfície hídrica que possui apenas $5 \%$ de chance de retornar no ano seguinte e assim sucessivamente. A quantidade de pixels também foi convertida em unidades de área para simplificar a análise dos resultados e optou-se por trabalhar com intervalos de frequência.

\section{RESULTADOS E DISCUSSÃO}

Os dados de superfície hídrica e precipitação para os estados do Ceará, Paraíba, Pernambuco e Rio Grande do Norte foram agregados com o propósito de verificar a relação existente entre a precipitação e a variação da superfície hídrica. Tais dados e suas respectivas tabulações cruzadas estão ilustrados na Figura 5. A partir da análise dos resultados, é possível identificar que o estado do Ceará possui a maior área de superfície hídrica, chegando a acumular duas vezes mais área de água superficial que os demais estados, além de registrar os maiores índices pluviométricos ao longo da

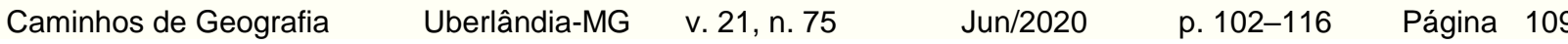


série temporal. Por outro lado, a Paraíba possui a menor área de superfície hídrica e os menores registros pluviométricos, seguida pelo Rio Grande do Norte e Pernambuco, respectivamente.

Quanto à proporção entre a extensão territorial dos estados e suas áreas de superfícies hídricas, o Rio Grande do Norte destaca-se com aproximadamente $2,4 \%$ de sua superfície composta por corpos hídricos, enquanto para Pernambuco essa mesma proporção é de aproximadamente $0,8 \%$. Essa proporção vai de encontro a capacidade de armazenamento dos estados, onde o Semiárido Potiguar possui uma capacidade de armazenamento de $4333 \mathrm{hm}^{3}$, enquanto o Semiárido Pernambucano armazena $2257 \mathrm{hm}^{3}$ de água (INSA, 2019).

Os dados de precipitação evidenciam o período de seca ocorrido a partir do ano de 2012, com a nítida diminuição dos valores de precipitação acumulada, fato que acarretou o declínio das superfícies hídricas nos referidos estados e a ocorrência de inúmeros conflitos políticos e sociais, alguns destes apontados por Marengo et al. (2016). Embora haja uma precipitação superior em 2017 em relação ao ano anterior no Ceará e Rio Grande do Norte, as precipitações ainda são abaixo do esperado e insuficientes para restituir o volume dos corpos hídricos, contribuindo para o agravamento da desertificação do Nordeste (TAVARES et al., 2019). Por isso, 2017 foi o ano de secas mais intensas registradas na plataforma Monitor de Secas para os estados em análise (ANA, 2019a).

Figura 5 - Relação da superfície hídrica e precipitação anual acumulada.
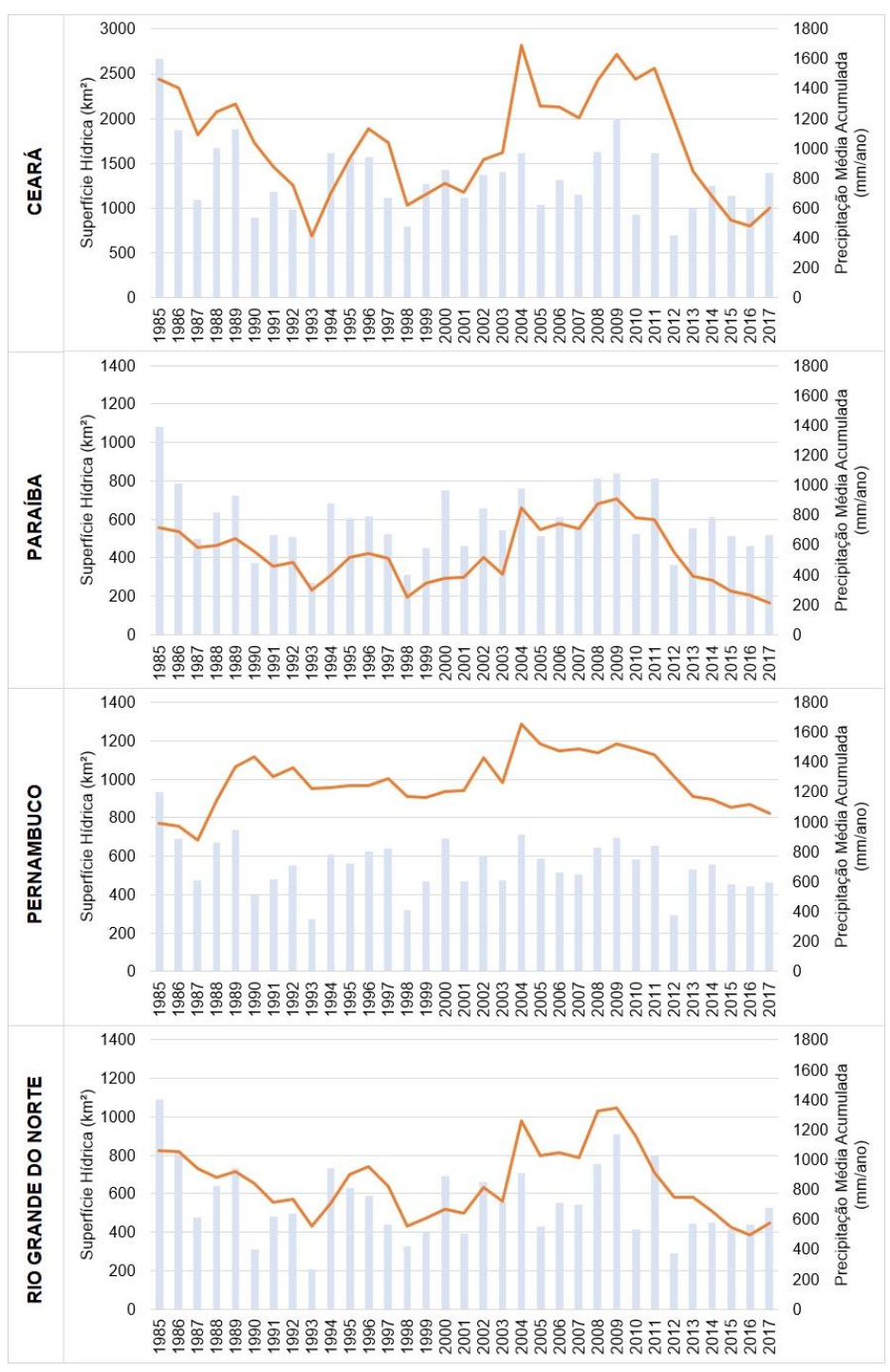

- Superfície Hídrica Precipitação

Fonte: Autoria própria. 
O estado do Ceará possui as condições mais favoráveis de oferta hídrica - maiores volumes de precipitação e maior área de superfície hídrica. Entretanto, encontra-se com menos de $16 \%$ de sua capacidade total de armazenamento (INSA, 2019), reflexo da alta demanda de água do Estado. Pois, segundo o Diagnóstico dos Serviços de Água e Esgoto, o Ceará possui o maior consumo de água per capita (126,70 litros/habitante/dia) entre os estados analisados (SNIS, 2018). Em contraposto, o estado de Pernambuco (mais populoso entre os analisados), possui um menor consumo per capita (96,70 litros/habitante/dia) e a passagem de um rio perene de grande porte em seu território, o Rio São Francisco, garantindo uma maior segurança hídrica ao estado.

Entretanto, até esse rio foi gravemente atingindo com a seca recente. Em 2017, o Rio São Francisco teve sua vazão reduzida ao menor nível dos últimos 38 anos. Esta situação impossibilitou a navegação de embarcações de grande porte em alguns locais e impactou diretamente a população ribeirinha, que depende do Rio para suas atividades de pesca e agricultura, principalmente na região do Baixo São Francisco, mais próximo da sua foz (G1, 2017).

A Paraíba atinge seu maior registro de superfície hídrica em 2009 com 707,62 km², com exceção de 1985 que registrou valores atípicos acima da média para todos os estados. Enquanto os demais ultrapassam frequentemente a marca de $800 \mathrm{~km}^{2}$. O estado também é detentor do menor registro, chegando a 167,04 $\mathrm{km}^{2}$ em 2017, evidenciando o colapso de diversos açudes e a consequente proibição da irrigação em diversas regiões, além de perdas críticas no setor agropecuário, conforme constatado por Rêgo et al. (2015). Esse mesmo autor também destaca a situação do Reservatório Epitácio Pessoa, um dos que se destacaram com os maiores impactos. A irrigação ao seu entorno foi suspensa e todas as cidades que eram abastecidas com suas águas passaram por racionamento no abastecimento de água pela concessionária. Dentre elas, a cidade de Campina Grande - segunda maior do estado e reconhecida pelo seu polo educacional e industrial, no período mais crítico, recebia água apenas dois dias por semana.

A análise da correlação estatística entre a superfície hídrica e a precipitação em todos os estados analisados (Figura 6), demonstra a variabilidade do comportamento das correlações ao passar dos anos em diferentes intervalos temporais, além da diferença notória do comportamento da variável entre os estados.

Figura 6 - Correlação entre superfície hídrica e precipitação acumulada.

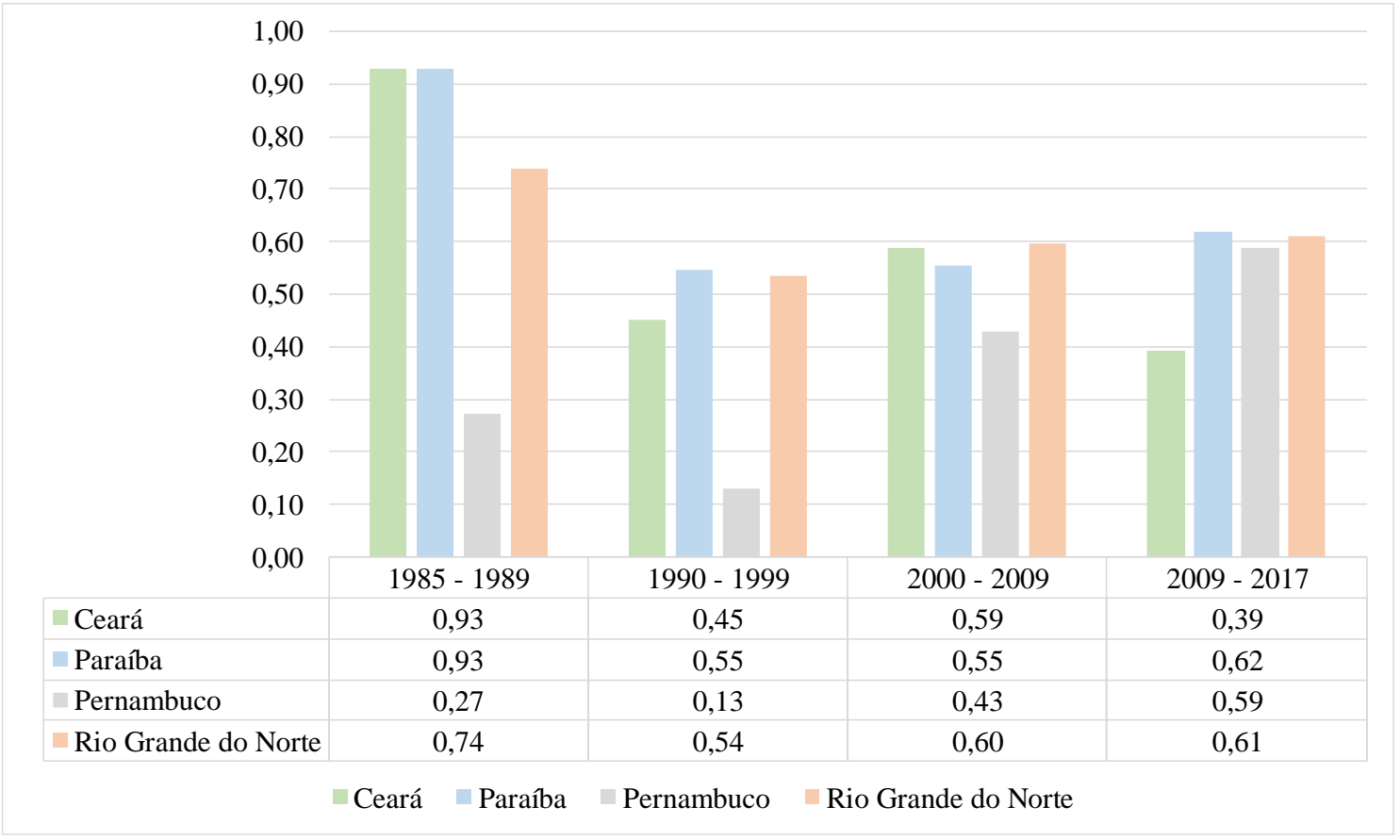

Fonte: Autoria própria. 
Análise temporal da disponibilidade hídrica nos estados beneficiados pela transposição do Rio São Francisco
Higor Costa de Brito

Yáscara Maia Araújo de Brito Wanessa Dunga de Assis

Yuciara Costa Barbosa Ferreira Rochele Sheila Vasconcelos lana Alexandra Alves Rufino

Com a análise da figura supramencionada, é possível identificar um crescimento da correlação nos estados da Paraíba, Pernambuco e Rio Grande do Norte a partir da década de 90. Vale salientar que entre os anos 80 e 90 o DNOCS construiu 36 açudes públicos na região Nordeste, aumentando a capacidade de reservação da região em 4.313,2 hectômetros cúbicos, caracterizando o maior acréscimo de volume armazenável do século para essa região (ASSUNÇÃO e LIVINGSTONE, 1993). Essas políticas ocasionaram aumentos significativos nas superfícies hídricas dos estados, acarretando em uma maior resposta ao regime pluviométrico, uma vez que novas áreas passam a ser inundáveis.

O Ceará, diferentemente dos demais estados, apresenta um comportamento arbitrário, registrando entre os anos de 2009 e 2017 uma correlação de 0,39, ou seja, a precipitação ocorrida no estado não reflete significativamente o aumento da superfície hídrica dos corpos d'água. Esse fato pode ser justificado através da grande capacidade de armazenamento superficial do estado e, uma vez que o mesmo permaneceu com uma superfície hídrica inferior a $1500 \mathrm{~km}^{2}$ a partir de 2011 , restituir todo o potencial hídrico da região torna-se um grande desafio e requer altos índices pluviométricos e políticas de gestão eficientes para que a demanda hídrica seja suprida e o balanço hídrico se torne positivo a ponto de aumentar a superfície hídrica do estado.

No estado de Pernambuco, a correlação decresce nos dois primeiros intervalos temporais, iniciando o crescimento entre o período de 2000 a 2009, chegando a uma correlação de 0,59 no último período, valor duas vezes maior que a inicial de 0,27 . Esse comportamento reflete o crescimento acentuado da dependência pluviométrica do estado, que anteriormente sofria de forma menos intensa aos eventos hidrológicos extremos. Tal constatação é preocupante, uma vez que o estado teve sua correlação aumentada progressivamente a partir da década de 90 , fato que pode ser ocasionado pela conversão de corpos hídricos perenes em intermitentes, afetando diretamente a segurança hídrica da região.

Diante da necessidade de analisar a perenidade dos corpos hídricos dos estados, os dados referentes a recorrência dos corpos hídricos (Tabela 2) demonstram a similaridade da recorrência da superfície hídrica ano após ano entre os estados. Através dessa análise também foi possível estimar a área em quilômetros quadrados dos espelhos d'água de cada estado.

Tabela 2 - Intervalos de recorrência dos corpos hídricos.

\begin{tabular}{cccccccccc}
\hline $\begin{array}{c}\text { Frequência com que a água } \\
\text { retorna de ano para ano (\%) }\end{array}$ & \multicolumn{2}{c}{ Ceará } & \multicolumn{3}{c}{ Paraíba } & \multicolumn{2}{c}{ Pernambuco } & \multicolumn{2}{c}{$\begin{array}{c}\text { Rio Grande do } \\
\text { Norte }\end{array}$} \\
& $\mathrm{km}^{2}$ & $\%$ & $\mathrm{~km}^{2}$ & $\%$ & $\mathrm{~km}^{2}$ & $\%$ & $\mathrm{~km}^{2}$ & $\%$ \\
\hline 0 a 25 & 77,00 & 3 & 29,28 & 4 & 31,49 & 4 & 51,31 & 4 \\
26 a 50 & 703,36 & 26 & 197,33 & 28 & 231,14 & 29 & 330,10 & 26 \\
51 a 75 & 990,50 & 36 & 262,75 & 38 & 274,72 & 35 & 445,99 & 35 \\
76 a 100 & 949,19 & 35 & 210,77 & 30 & 254,89 & 32 & 437,36 & 35 \\
\hline Total & $2.720,03$ & 100 & 700,13 & 100 & 792,25 & 100 & $1.264,76$ & 100 \\
\hline \multicolumn{8}{c}{ Fonte: Autoria própria. }
\end{tabular}

É notória a similaridade dos percentuais nos intervalos de frequência entre cada estado, nos quais cerca de $33 \%$ das superfícies tendem a ser restituídas a cada ano. Entretanto, se essa análise for feita com base na mediana, é possível constatar que aproximadamente $31 \%$ das áreas hídricas possuem menos de $50 \%$ de chance de serem restituídas de um ano para o outro, indicando a alta variabilidade da disponibilidade hídrica dos estados.

A partir de uma análise quantitativamente dos valores de superfície hídrica, temos que o Ceará possui uma área de aproximadamente $780 \mathrm{~km}^{2}$ com chance inferior a $50 \%$ de retornar de um ano para o outro, ou seja, o estado pode perder uma área de superfície hídrica equivalente a toda superfície contida no estado da Paraíba em apenas um ano. Esse fato reforça a discussão feita com base na correlação entre a pluviometria e a área de superfície hídrica, uma vez que diante da dimensão, torna-se um desafio restituir uma superfície hídrica tão extensa.

Caminhos de Geografia $\quad$ Uberlândia-MG $\quad$ v. 21, n. 75 $\quad$ Jun/2020 $\quad$ p. 102-116 Página 112 
Diante da dimensão das superfícies hídricas dos estados, vale salientar que a perda de água por evaporação também é um fator diretamente influenciado pela dimensão dos espelhos d'água. Nesse contexto, o Ceará e o Rio Grande do Norte seriam os estados com um maior potencial de evaporação dentre os analisados. Será q dava pra colocar as taxas de evapotranspiração?

\section{CONSIDERAÇÕES FINAIS}

A disponibilidade gratuita de produtos gerados a partir de técnicas de sensoriamento remoto permitem análises quali-quantitativas de todos os corpos hídricos presentes no globo terrestre e devem ser levadas em consideração pelos órgãos gestores nas tomadas de decisão, uma vez que esses produtos evidenciam as particularidades de cada localidade. Os resultados obtidos demonstram a vulnerabilidade hídrica dos estados beneficiados com a transposição do Rio São Francisco - os que detém maior superfície hídrica não necessariamente proporcionam uma maior segurança hídrica à população. Com isso, fica evidente a necessidade de repensar a ideia de que o aumento da oferta de água por meio de construção de barragens e canais são a única solução para garantir o acesso à água.

As séries históricas de precipitação e superfície hídrica demonstram que a seca hidrológica é um fenômeno recorrente no semiárido brasileiro. Dessa forma, a gestão e a governança da água nessas regiões necessitam de um menor caráter imediatista em virtude da crescente demanda hídrica. Cenários prospectivos devem ser levados em consideração em obras de infraestrutura e políticas públicas.

A transposição do Rio São Francisco foi planejada e implantada como uma alternativa às constantes crises hídricas ocorridas na região Nordeste. Entretanto, o crescimento populacional desenfreado e o constante aumento do consumo, direto ou indireto, comprometem essa promessa, visto que a própria bacia do Rio sofre com a seca e a obra pode proporcionar aos gestores uma sensação de segurança hídrica nos estados beneficiados. Medidas de gestão da demanda efetivas carecem de ser tomadas para garantir o desenvolvimento sustentável da região, de forma a não comprometer a disponibilidade hídrica do Rio São Francisco, evitando assim que uma obra desse porte se torne rapidamente obsoleta e ineficaz.

O presente estudo apresentou inúmeras análises derivadas a partir de dados de superfície hídrica. Nesse aspecto, pesquisas futuras que levem em consideração as variações dos volumes de água armazenada em cada estado podem aprimorar tais análises e fortalecer as discussões acerca do tema. Além disso, esse estudo pode ser realizado em escalas menores, tornando factível uma avaliação mais precisa em locais onde possa haver maiores conflitos pelo uso e disponibilidade de água, resultando em uma ferramenta promissora para subsidiar políticas públicas em locais de maior vulnerabilidade hídrica.

\section{AGRADECIMENTOS}

Os autores agradecem a Coordenação de Aperfeiçoamento de Pessoal de Nível Superior (CAPES), ao Conselho Nacional de desenvolvimento Científico e Tecnológico (CNPq) e à Fundação de Apoio à Pesquisa do Estado da Paraíba (FAPESQ) pela concessão de bolsas aos pesquisadores envolvidos.

\section{REFERÊNCIAS}

ALSDORF, D. E.; RODRíGUEZ, E.; LETTENMAIER, D. P. Measuring surface water from space.

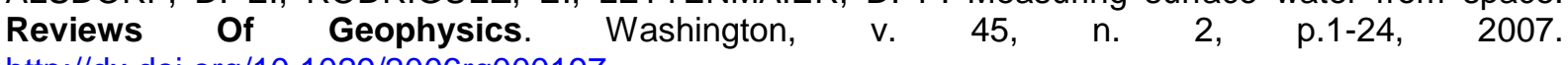
http://dx.doi.org/10.1029/2006rg000197

ALVALÁ, R. C. S.; CUNHA, A. P. M. A.; BRITO, S. S. B.; SELUCHI, M. E.; MARENGO, J. A.; MORAES, O. L. L; CARVALHO, M. A. Drought monitoring in the Brazilian Semiarid region. Anais da Academia Brasileira de Ciências. Rio de Janeiro, v. 91 (suppl 1), p. 2-15, 2017. https://doi.org/10.1590/0001-3765201720170209

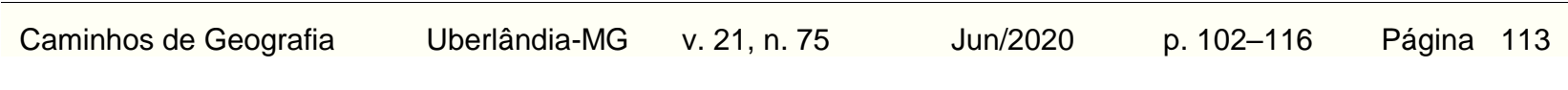


AMORIM, A. L.; RIBEIRO, M. M. R.; BRAGA, C. F. C. Conflitos em bacias hidrográficas compartilhadas: o caso da bacia do rio Piranhas-Açu/PB-RN. Revista Brasileira de Recursos Hídricos. Porto Alegre, v. 21, n. 1, p. 36-45, 2016. https://doi.org/10.21168/rbrh.v21n1.p36-45

ANA - Agência Nacional das Águas. Conjuntura dos recursos hídricos no Brasil 2017: relatório pleno. Brasília-DF: ANA, 2017. Disponível em : http://www.snirh.gov.br/portal/snirh/ centrais-deconteudos/conjuntura-dos-recursos-hidricos/conj2017_rel-1.pdf Acesso em: 10 de julho 2019.

Portal do Monitor de Secas. 2019a. Disponível em: http://monitordesecas.ana.gov.br/ Acesso em: 16 de julho de 2019a.

Base Hidrográfica Ottocodificada Multiescalas. 2019b. Disponível em: https://metadados.ana.gov.br/geonetwork/srv/pt/metadata.show?id=586\&currTab=distribution Acesso em: 12 de outubro de 2019.

ASSUNÇÃO, L. M.; LIVINGSTONE, I. Desenvolvimento inadequado: construção de açudes e secas no sertão do Nordeste. Revista Brasileira de Economia. Rio de Janeiro, v. 3, n. 47, p.425-448, 1993. Disponível em: <http://bibliotecadigital.fgv.br/ojs/index.php/rbe/article/viewFile/582/7932>.

BRASIL. Projeto de Integração do Rio São Francisco com Bacias Hidrográficas do Nordeste Setentrional: Relatório de Impacto Ambiental - RIMA. Brasil: Ministério da Integração Nacional, 2004. Disponível em: http://www.mi.gov.br/ documents/10157/3675235/ RIMA+JULHO+2004.pdf/78989068cf76-4ab5-bf01-3b45473db7f9 Acesso em: 25 de janeiro de 2019.

CAMPOS, J. N. B. Secas e políticas públicas no semiárido: ideias, pensadores e períodos. Estudos Avançados. São Paulo, v. 28, n. 82, p. 65-88, 2014. https://doi.org/10.1590/S0103$\underline{40142014000300005}$

COELHO, C. A. S.; UVO, C. B.; AMBRIZZI, T. Exploring the impacts of the tropical Pacific SST on the precipitation patterns over South America during ENSO periods. Theoretical and Applied Climatology. Hamburg, v. 71, n. 3-4, p. 185-197, 2002. https://doi.org/10.1007/s007040200004

CRAUSBAY, S. D. RAMIREZ, A. R. Defining Ecological Drought for the Twenty-First Century. American Meteorological Society. Boston, v. 98, n. 12, p. 2543-2550, 2017. https://doi.org/10.1175/BAMS-D-16-0292.1

CUNHA, A. M. P. A.; BRITO, S. S. B.; ROSSATO, L.; ALVALÁ, R. C. S.; CARVALHO, M. A.; ZERI, M.; CUNNINGHAM,C.; MACIEL, A. P. R.; ANDRADE, E. S.; VIEIRA, R. M. S. P. Avaliação de indicador para o monitoramento dos impacos da seca em áreas de pastagens no Semiárido do Brasil. Revista Brasileira de Cartografia. Monte Carmelo, v. 69, n. 1, p. 89-106, 2017. Disponível em: $<$ http://www.seer.ufu.br/index.php/revistabrasileiracartografia/article/view/44034>.

DE SOUZA, E. B.; KAYANO, M. T.; AMBRIZZI, T. Intraseasonal and submonthly variability over the Eastern Amazon and Northeast Brazil during the autumn rainy season. Theoretical and Applied Climatology. Hamburg, v. 81, n. 3-4, p. 177-191, 2005. https://doi.org/10.1007/s00704-004-0081-4

FUNK, C.; PETERSON, P.; LANDSFELD, M.; PEDREROS, D.; VERDIN, J.; SHUKLA, S. HUSAK, G.; ROWLAND, J.; HARRISON, L.; HOELL, A.; MICHAELSEN, J. The climate hazards infrared precipitation with stations - a new environmental record for monitoring extremes. Sci Data 2. London, 150066, 2015. https://doi.org/10.1038/sdata.2015.66

G1 - Portal de Notícias G1 da Rede Globo de Televisão. Rio São Francisco tem vazão reduzida ao menor nível em 38 anos. 2017. Disponível em: http://g1.globo.com/jornalnacional/noticia/2017/07/rio-sao-francisco-tem-vazao-reduzida-ao-menor-nivel-em-38-anos.html Acesso em: 4 de março de 2020.

GORELICK, N.; HANCHER, M.; DIXON, M.; ILYUSHCHENKO, S. THAU, D.; MOORE, R. Google Earth Engine: Planetary-scale geospatial analysis for everyone. Remote Sensing of Environment. Toronto, v. 202, p. 18-27, 2017. https://doi.org/10.1016/i.rse.2017.06.031

GRIMM, A. M. The El Niño Impact on the Summer Monsoon in Brazil: Regional Processes versus Remote Influences. Journal of Climate. Boston, v. 16, n. 2, p. 263-280, 2003. https://doi.org/10.1175/1520-0442(2003)016\%3C0263:TENIOT\%3E2.0.CO;2 
GRIMM, A. M. How do La Niña events disturb the summer monsoon system in Brazil?. Climate Dynamics. New York, v. 22, n. 2-3, p. 123-138, 2004. https://doi.org/10.1007/s00382-003-0368-7

IBGE - Instituto Brasileiro de Geografia e Estatística. Downloads. 2017a. Disponível em: https://www.ibge.gov.br/geociencias/downloads-geociencias.html Acesso em: 12 de outubro de 2019. de 2019 .

IBGE - Cidades. 2017b. Disponível em: https://cidades.ibge.gov.br/ Acesso em: 1 de agosto

INSA - Instituto Nacional do Semiárido. Olho n'água. 2019. Disponível em: https://olhonagua. insa.gov.br/ Acesso em: 2 de julho de 2019.

MAISONNAVE, F.; PRADO, A. Nordeste: Seca histórica já dura seis anos e ameaça tornar-se regra no semiárido. Jornal Folha de São Paulo (On-line). Publicado em 24 de abril de 2018. Disponível em: https://arte.folha.uol.com.br/ciencia/2018/ crise-do-clima/nordeste/seca-historica-ja-dura-seis-anos-eameaca-tornar-se-regra-no-semiarido/ Acesso em: 25 de janeiro de 2019.

MARENGO, J. A.; CUNHA, A. P.; ALVES, L. M. A seca de 2012-15 no semiárido do Nordeste do Brasil no contexto histórico. Climanálise. São Carlos, v. 3, p. 49-54, 2016. Disponível em: http://climanalise.cptec.inpe.br/ rclimanl/revista/pdf/30anos/marengoetal.pdf Acesso em 10 de maio de 2019.

MARTINS, E. S. P. R.; DE NYS, E.; MOLEJÓN, C.; BIAZETO, B.; SILVA, R. F. V.; ENGLE, N. Monitor de Secas do Nordeste, em busca de um novo paradigma para a gestão de secas. $1^{\text {a }}$ Edição. Brasília: Banco Mundial, 2015. Disponível em: http://www.worldbank.org/pt/country/ brazil/brief/brazil- Acesso em: 4 de julho de 2019.

MCKEE, T. B.; DOESKEN, N. J; KLEIST, J. The relationship of drought frequency and duration to time scales. In: CONFERENCE ON APPLIED CLIMATOLOGY, 8., 1993. Proceedings... Anaheim: Boston, 1993. p. 179 - 183. Disponível em: http://www.droughtmanagement.info/ literature/AMS_Relationship_Drought_Frequency_Duration_Time_Scales_1993.pdf Acesso em: 2 de agosto de $201 \overline{9}$.

MEDEIROS, F. J; LIMA, K. C.; CAETANO, D. A.; SILVA, F. J. O. Impacto da Variabilidade Interanual da Precipitação nos Reservatórios do Semiárido do Nordeste do Brasil. Anuário do Instituto de $\begin{array}{lllllll}\text { Geociências. Rio de Janeiro, v. } 41, \quad \text { n. } 3, & \text { p. } & 731-741,\end{array}$ http://dx.doi.org/10.11137/2018 3731741

MONTENEGRO, A. A. A.; MONTENEGRO, S. M. G. L. Olhares sobre as políticas públicas de recursos hídricos para o semiárido. In: GHEYl, H. R. et al. (Eds.). Recursos Hídricos em Regiões Semiáridas: Estudos e Aplicações. Campina Grande - PB/Cruz das Almas - BA: Instituto Nacional do Semiárido/Universidade Federal do Recôncavo da Bahia, 2012. p. 2-6.

NORMANDIN, C.; FRAPPART, F.; LUBAC, B.; BÉLANGER, S.; MARIEU, V.; BLAREL, F. et al. Quantification of surface water volume changes in the Mackenzie Delta using satellite multi-mission data. Hydrology and Earth System Sciences. Göttingen, v. 22, n. 2, p. 1543-1561, 2018. https://doi.org/10.5194/hess-22-1543-2018

PARAÍBA (Estado). Decreto $\mathrm{n}^{\circ}$ 38.195, de 02 de abril de 2018. Disponível em: http://static.paraiba.pb. gov.br/2018/04/Diario-Oficial-03-04-2018.pdf Acesso em: 23 de agosto de 2019.

PAREDES-TREJO, F. J.; BARBOSA, H. A.; LAKSHMI KUMAR, T. V. Validating CHIRPS-based satellite precipitation estimates in Northeast Brazil. Journal of Arid Environments. Amsterdam, v. 139, p. 26-40, 2017. https://doi.org/10.1016/j.jaridenv.2016.12.009

PEKEL, J. F.; COTTAN, A.; GORELICK, N.; BELWARD, A. S. High-resolution mapping of global surface water and its long-term changes. Nature. Berlin, v. 540, n. 7633, p. 418-422, 2016. https://doi.org/10.1038/nature20584

RÊGO, J. C.; GALVÃO, C. O.; RIBEIRO, M. M. R.; ALBUQUERQUE, J. P. T.; NUNES, T. H. C. A Crise do Abastecimento de Campina Grande: Atuações dos Gestores, Usuários, Poder Público, Imprensa e População. In: Simpósio Brasileiro de Recursos Hídricos, 21, 2015. Anais... Brasília: ABRH, 2015. Disponível em: http://www.evolvedoc.com.br/sbrh/detalhes-1102_a-crise-do- 
abastecimento-de-campina-grande-atuacoes-dos-gestores-usuarios-poder-publico-imprensa-epopulacao Acesso em: 13 de julho de 2019.

SHUKLA, S.; WOOD, A. W. Use of a standardized runoff index for characterizing hydrologic drought. Geophysical Research Letters. Washington, v. 35, n. 2, p. 1-7, 2008. https://doi.org/10.1029/2007GL032487

SMITH, L. C. Satellite remote sensing of river inundation area, stage, and discharge: a review. Hydrological Processes. Hoboken, v. 11, n. 10, p.1427-1439, 1997. http://dx.doi.org/10.1002/(sici)1099-1085(199708)11:103.0.co;2-s

SNIS - Sistema Nacional de Informações sobre Saneamento. Diagnóstico dos Serviços de Água e Esgoto. Brasília: Ministério do Desenvolvimento Regional, 2018. 186 p. Disponível em: http://www.snis.gov.br/diagnostico-anual-agua-e-esgotos/diagnostico-dos-servicos-de-agua-eesgotos-2018 Acesso em: 05 de março de 2020.

SOUZA, C.; AZEVEDO, T. MapBiomas General Handbook. São Paulo: MapBiomas, 2017.

SUDENE - Superintendência do Desenvolvimento do Nordeste. Delimitação do Semiárido.2017. Disponível em: http://www.sudene.gov.br/delimitacao-do-semiarido Acesso em: 12 de outubro de 2019.

TANG, Q.; GAO, H.; LU, H.; LETTENMAIER, D. P. Remote sensing: hydrology. Progress in Physical Geography: Earth and Environment. Thousand Oaks, v. 33, n. 4, p. 490-509, 2009. https://doi.org/10.1177\%2F0309133309346650

TAVARES, V. C.; ARRUDA, Í. R. P.; SILVA, D. G. Desertificação, mudanças climáticas e secas no Semiárido brasileiro: uma revisão bibliográfica. Geosul. Florianópolis, v. 34, n. 70, p. 385-405, 2019. https://doi.org/10.5007/2177-5230.2019v34n70p385

TIMMERMANN, A., AN, S., KUG, J. et al. El Niño-Southern Oscillation complexity. Nature. Berlin, v. 559, n. 7715, p. 535-545, 2018. https://doi.org/10.1038/s41586-018-0252-6

VICENTE-SERRANO, S. M.; BEGUERÍA, S.; LÓPEZ-MORENO, J. I. A Multiscalar Drought Index Sensitive to Global Warming: The Standardized Precipitation Evapotranspiration Index. Journal of Climate. Boston, v. 23, n. 7, p. 1696-1718, 2010. https://doi.org/10.1175/2009JCLI2909.1

WANG, X.; XIE, H. A Review on Applications of Remote Sensing and Geographic Information Systems (GIS) in Water Resources and Flood Risk Management. Water. Basel, v. 10, n. 5, p. 608, 2018. https://doi.org/10.3390/w10050608

WENG, Q. Land use change analysis in the Zhujiang Delta of China using satellite remote sensing, GIS and stochastic modelling. Journal of Environmental Management. Amsterdam, v. 64, n. 3, p. 273-284, 2002. https://doi.org/10.1006/jema.2001.0509

WILHITE, D. A. Breaking the Hydro-Illogical Cycle: Changing the Paradigm for Drought Management. Earth Magazine. Alexandria, v. 57, n. 7, p. 71-72, 2012. Disponível em: https://digitalcommons.unl.edu/droughtfacpub/53/ Acesso em 30 de junho de 2019.

Recebido em: 24/09/2019

Aceito para publicação em: 12/03/2020 